\title{
Hippocampal CA1/subiculum-prefrontal cortical pathways induce plastic changes of nociceptive responses in cingulate and prelimbic areas
}

\author{
Hiroyuki Nakamura, Yoko Katayama, Yoriko Kawakami*
}

\begin{abstract}
Background: Projections from hippocampal CA1-subiculum (CA1/SB) areas to the prefrontal cortex (PFC), which are involved in memory and learning processes, produce long term synaptic plasticity in PFC neurons. We examined modifying effects of these projections on nociceptive responses recorded in the prelimbic and cingulate areas of the PFC.

Results: Extracellular unit discharges evoked by mechanical noxious stimulation delivered to the rat-tail and field potentials evoked by a single stimulus pulse delivered to CA1/SB were recorded in the PFC. High frequency stimulation (HFS, $100 \mathrm{~Hz}$ ) delivered to CA1/SB, which produced long-term potentiation (LTP) of field potentials, induced long-term enhancement (LTE) of nociceptive responses in $78 \%$ of cases, while, conversely, in $22 \%$ responses decreased (long-term depression, LTD). These neurons were scattered throughout the cingulate and prelimbic areas. The results obtained for field potentials and nociceptive discharges suggest that CA1/SB-PFC pathways can produce heterosynaptic potentiation in PFC neurons. HFS had no effects on Fos expression in the cingulated cortex. Low frequency stimulation (LFS, $1 \mathrm{~Hz}, 600$ bursts) delivered to the CA1/SB induced LTD of nociceptive discharges in all cases. After recovery from LTD, HFS delivered to CA1/SB had the opposite effect, inducing LTE of nociceptive responses in the same neuron. The bidirectional type of plasticity was evident in these nociceptive responses, as in the homosynaptic plasticity reported previously. Neurons inducing LTD are found mainly in the prelimbic area, in which Fos expression was also shown to be inhibited by LFS. The electrophysiological results closely paralleled those of immunostaining. Our results indicate that CA1/SB-PFC pathways inhibit excitatory pyramidal cell activities in prelimbic areas.

Conclusion: Pressure stimulation (300 g) applied to the rat-tail induced nociceptive responses in the cingulate and prelimbic areas of the PFC, which receives direct pathways from CA1/SB. HFS and LFS delivered to the CA1/SB induced long-term plasticity of nociceptive responses. Thus, CA1/SB-PFC projections modulate the nociceptive responses of PFC neurons.
\end{abstract}

\section{Background}

The two segregated central pathways for sensory-discriminative and affective dimensions of pain have been examined in human brain imaging studies [1], which indicated that neural activities of the prefrontal cortex (PFC) participate in the affectional dimension of pain $[2,3]$. Basic lesion studies in animals have implicated the PFC as the center of the pain-related fear emotion [4], which involves affective responses to noxious and fear

\footnotetext{
* Correspondence: blackcat@research.twmu.ac.jp

Department of Physiology, Graduate School of Medicine, Tokyo Women's Medical University, 8-1 Kawada-cho, Shinjuku-ku, Tokyo 162-8666, Japan
}

stimuli [5]. The PFC also has a possible role in the execution and storage of long-term memory [6]. Using PFC slices, high frequency stimulation (HFS) delivered to layer II of the PFC induced long-term depression (LTD) or potentiation (LTP) in neurons of layer V [7]. HFS delivered to $C A 1$ regions induced NMDA (N-Methyl-D-Aspartate)-mediated LTP in the PFC [8], indicating CA1-PFC pathways to be the site of postsynaptic excitatory potentiation.

CA1 pyramidal cells in the hippocampus (HP) receive pain information from peripheral nociceptors [9]. In a rat in vivo study, CA1 pyramidal cells were depressed by

\section{()


intense noxious stimulation applied to the tail [10]. Human brain imaging analysis showed that noxious laser stimulation evoked pain responses in the HP [11]. Responses to noxious heat stimulation in the bilateral HP were markedly increased in an anxious state as compared to circumstances not associated with anxiety [12]. These reports suggest that HP might be involved in the affectional dimension of pain.

The projections from the HP to the PFC were established by anatomical $[13,14]$ and physiological $[15]$ studies. HP projections terminate on spiny pyramidal neurons of the PFC and form excitatory synapses [16]. Excitatory unit responses evoked by CA1/SB stimulation were identified in the prelimbic area [17]. Moreover, approximately $70 \%$ of prelimbic interneurons were activated by direct projections from the HP, which induced feed forward inhibition of pyramidal cells [18]. The synapses of hippocampal fibers in the PFC can express different forms of plasticity. The direct excitatory glutamate pathways from the CA1/SB to the PFC [19] are related to NMDA receptor mediated LTP [8]. LTD of field potentials was also induced in HP-PFC pathways by low frequency burst stimulation (LFS) [20]. HP-PFC pathways have been suggested to be required for working memory $[21,22]$. In an animal behavioral study, bilateral inhibition of the PFC reportedly disrupted spatial working memory [23]. HP-PFC pathways may be related to higher mnemonic functions of pain (e.g. fear conditioned leaning).

We analyzed the effects of CA1/SB inputs into the prelimbic and cingulate areas of the PFC on nociceptive responses evoked by peripheral mechanical noxious stimulation. HFS/LFS delivered to the CA1/SB induced LTP/LTD-like changes in nociceptive responses recorded in the PFC, suggesting the HP-PFC pathway to be involved in affectional memory in pain processing.

\section{Methods}

\section{Animal preparation}

Adult male Wistar rats (280 350 g: Sankyo Laboratory Co, Tokyo, Japan) were used in all experiments. The rats were housed under controlled temperature $\left(25^{\circ} \mathrm{C}\right)$ and humidity (40-50\%) conditions with a 12-h light/ dark cycle, and had free access to food and water. Experiments conformed to guidelines issued by the National Institutes of Health for Laboratory Animals and all procedures were approved by the Animal Experimental Committee of Tokyo Women's Medical University. Efforts were made to minimize the number of animals used and their suffering. All rats were anesthetized with a single injection of urethane $(1.5 \mathrm{~g} / \mathrm{kg}$, i.p.) and mounted in a stereotaxic instrument (Narisige, Tokyo, Japan). Body temperature was maintained at 37 $38^{\circ} \mathrm{C}$ using a chemical thermo-mat.

\section{Recording of extracellular discharges}

Recording electrodes (stainless steel 9 - $12 \mathrm{M} \Omega$, USA) were placed in the cingulate or prelimbic areas of the PFC (coordinates: $3.2 \mathrm{~mm}$ anterior and $0.5 \mathrm{~mm}$ lateral to the bregma, Fig. 1). The multi or single unit spikes were processed with a multichannel amplifier (MEG6100; Nihon Koden Co., Tokyo, Japan) and an active filter $(500 \mathrm{~Hz}-3 \mathrm{KHz}, \mathrm{DV}-04$, NF Electronic Instruments Co.Tokyo, Japan). Data were sent to a personal computer (Macintosh G4; Apple Co., Tokyo, Japan) via an integrated system (Power Lab/4SP; Mountain View, CA, USA) for recording storage and later off-line analysis. A single unit spike was discriminated on the basis of the height and width of each unit from a multi-unit recording (cluster analysis, Fig. 2A D) obtained with software Chart 4.1 (AD Instruments Co., Tokyo, Japan). Single unit discrimination from multi-units is presented on the third line of Fig $2 \mathrm{~A} \sim \mathrm{C}$. The spike histogram was analyzed using clusters of single spikes. Each bin of histograms consists of spikes recorded during a $500 \mathrm{~ms}$ or $1000 \mathrm{~ms}$ period (Fig 2E).

\section{Mechanical stimulation}

We applied mechanical pressure to the rat-tail, at $1.0-2.0$ $\mathrm{cm}$ distal to the body, using a mechanical stimulator (DPS270 DIA Medical System Co. Tokyo, Japan), equipped with a probe with a circular contact area and a $1 \mathrm{~mm}$ in diameter tip. Mechanical stimuli were delivered every $90 \mathrm{~s}$ at constant force with a feedback system. Stimulus intensities used in this experiment were 300 - 500 gf with a $0.1 \mathrm{~s}$ rising (and decreasing) time to maximum force and a $2 \mathrm{~s}$ hold time. The stimulus condition applied to the tail evoked c-fiber activities in peripheral nerves [24].

\section{Electrical Simulation}

In these experiments, a monopolar stainless steel stimulus electrode (stainless steel 1-3 M $\Omega$, USA) was lowered into the dorsal portion of the CA1/SB area (coordinates: 4.8 5.3 $\mathrm{mm}$ posterior and $2.0-3.2 \mathrm{~mm}$ lateral to the bregma, and $1.8-3.6 \mathrm{~mm}$ in depth from the surface Fig 1). HFS $(100 \mathrm{~Hz}$ frequency, $250 \mu$ s duration, $20 \mu \mathrm{A}$ intensity pulse) of $15 \mathrm{sec}$ duration was delivered as a conditioning stimulation $(\mathrm{n}=27)$. LFS was delivered in 600 bursts $(1 \mathrm{~Hz}, 100 \mu \mathrm{A}, 5$ pulses $)$. Unit discharges evoked by nociceptive stimulation were recorded every $90 \mathrm{~s}$ and the last three data points served as pre-HFS/ LFS control values. The stability of responses for at least 20 min before pre-control recording was confirmed.

\section{Field potential recording in preliminary experiments}

In the preliminary experiments, we confirmed that the stimulus condition for HFS used in our experiments induced LTP (Fig 3). Field potentials evoked by CA1/SB stimulation (single pulse, $250 \mu$ s duration, $100-140 \mu \mathrm{A}$ ) 


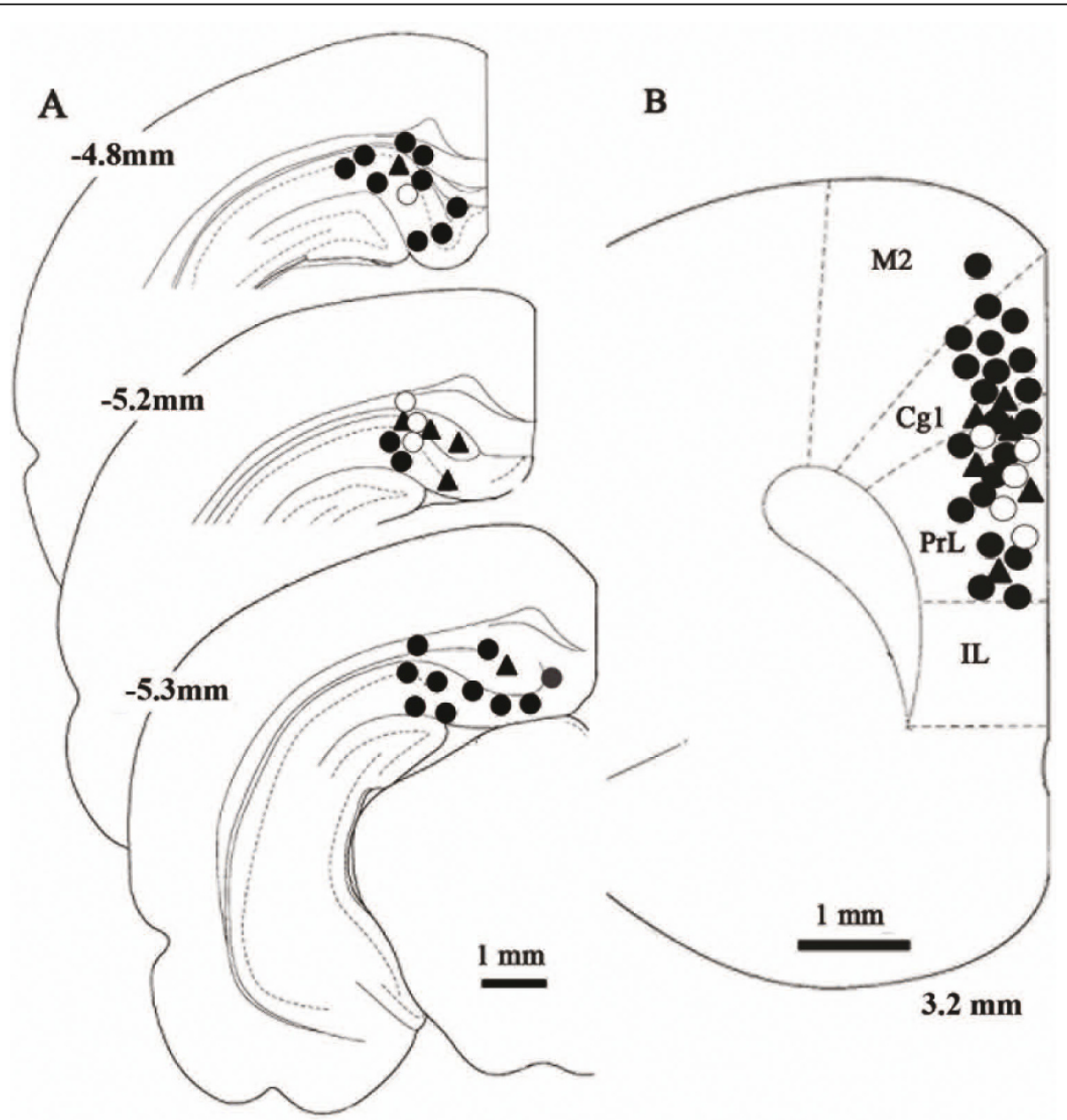

Figure 1 Locations of stimulus and recording sites. A: Stimulus locations in CA1/subiculum areas. Numbers on the left represent distance from the bregma. B: Recording sites in the PFC. Solid circles represent the locations of neurons in which LTE was induced by HFS. Triangles represent LTD induced by HFS. Open circles are neurons in which LTD was induced by LFS. The number represents distance from the bregma. M2: secondary motor cortex, Cg1:cingulate cortex area 1, PrL: prelimbic cortex, IL: infralimbic cortex.

were recorded $(0.5 \mathrm{~Hz}-30 \mathrm{KHz})$ before and after HFS delivery to the CA1/SB in the PFC. The PFC field potentials evoked by stimulation applied to CA1/SB, which contained $6 \mathrm{~ms}$ peak latency positive waves (P6) and 16 ms latency negative waves (N16), were recorded in the PFC (5 animals). LTP of these field potentials was induced by HFS delivered to the CA1/SB and persisted for about $60 \mathrm{~min}$. The maximum increase in amplitude $(144.1 \% \pm 23, \mathrm{p}<0.05)$ was observed at $30 \mathrm{~min}$ after HFS, while values at 60,90 and 120 min were $143 \pm 17 \%$ ( $\mathrm{p}<0.05), 119 \pm 13 \%$ and $100 \pm 4 \%$, respectively. The HFS used in our experiments confirmed LTP of field potentials, as demonstrated by previous studies $[17,25]$.

\section{Locations of unit recording and stimulus electrodes}

The locations of units and stimulus points were marked with a positive electric current lesion (direct current, 80 $\mu \mathrm{A}$ for $10 \mathrm{~s}$ ). At the end of each experiment, the animals were perfused with normal saline and $4 \%$ paraformaldehyde. After overnight post-fixation, the brains were sectioned $(50 \mu \mathrm{m})$ and stained with hematoxylin-eosin solution for examination of the recording and stimulus sites under light microscopy.

We counted the number of Fos-positive cells in the PFC [26] with HFS (5 control animals and 5 animals with HFS) and LFS (5 control animals and 5 animals with LFS). All animals were perfused with $4 \%$ paraformaldehyde one hour after conditioning stimulation delivered to the HP, followed by Fos staining. The brains were left in $10 \%$ sucrose overnight and then stored frozen. The frozen brain tissues were sectioned at $10 \mu \mathrm{m}$ (Cryostat CM1850, Leica) and incubated with anti-c-Fos antibody (x 10000, Ab-5, Oncogene, CatnoPC38) overnight at $4^{\circ} \mathrm{C}$. Numbers of Fos positive cells in $400 \mu \mathrm{m}^{2}$ areas in the cingulate and prelimbic areas were counted in 15 slices for each animal. 
A

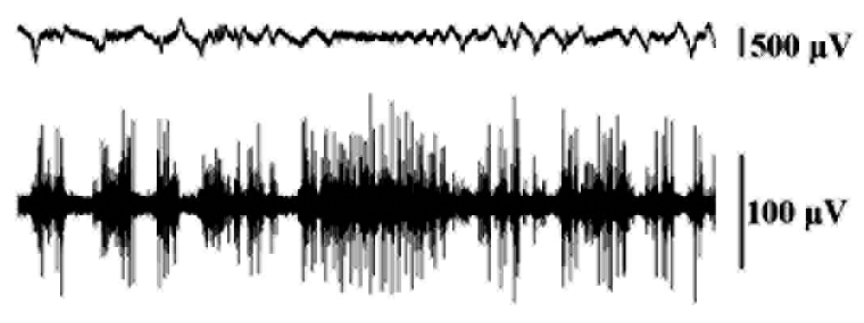

response
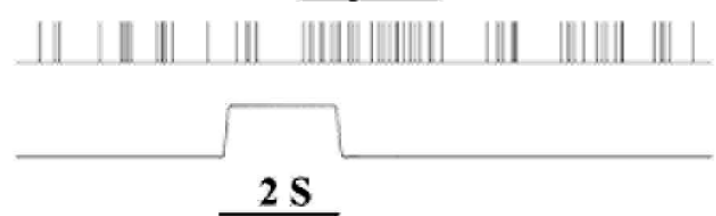

|500 g

B
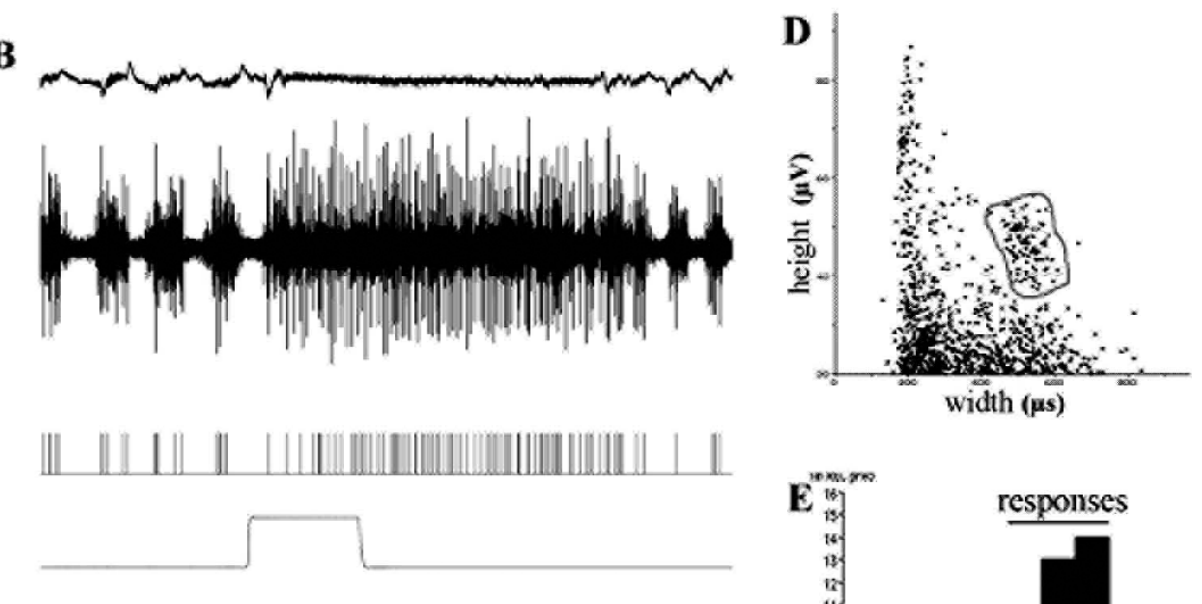

C
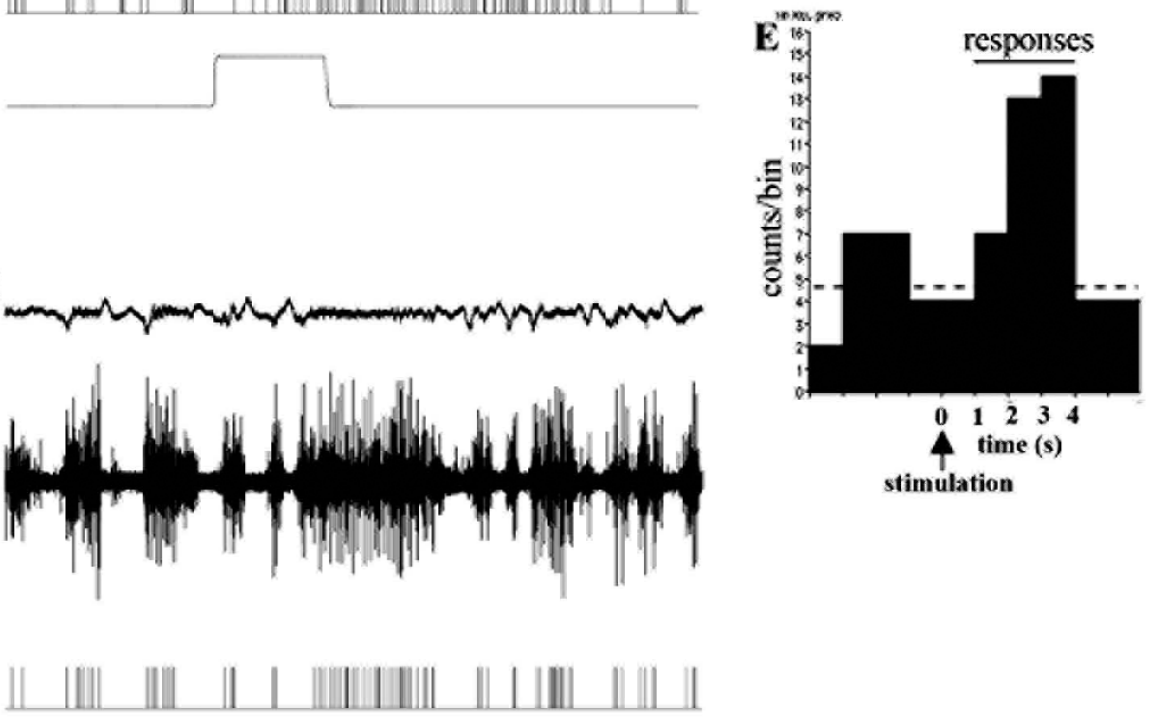

Figure 2 Effects of CA1/SB inputs on nociceptive responses recorded in the PFC. A: Nociceptive responses evoked by mechanical stimulation. The top trace represents an electrocorticogram (ECo). The second trace is multiple unit discharges evoked by mechanical stimulation (500 g pressure bottom trace). The third trace represents single unit responses selected by cluster analysis from multi-units in the second line. The bottom trace represents a pressure curve. B: 30 min after HFS delivery to the subiculum areas of the HP, the discharge durations of nociceptive neurons were increased. C: 90 min after HFS nociceptive responses had recovered to the pre-control level. D: Discriminator view obtained using Spike Histogram software. Spikes were selected with the gray line representing single responses. E: Spike histogram composed of the spikes selected above. One bin is $1 \mathrm{~s}$. 


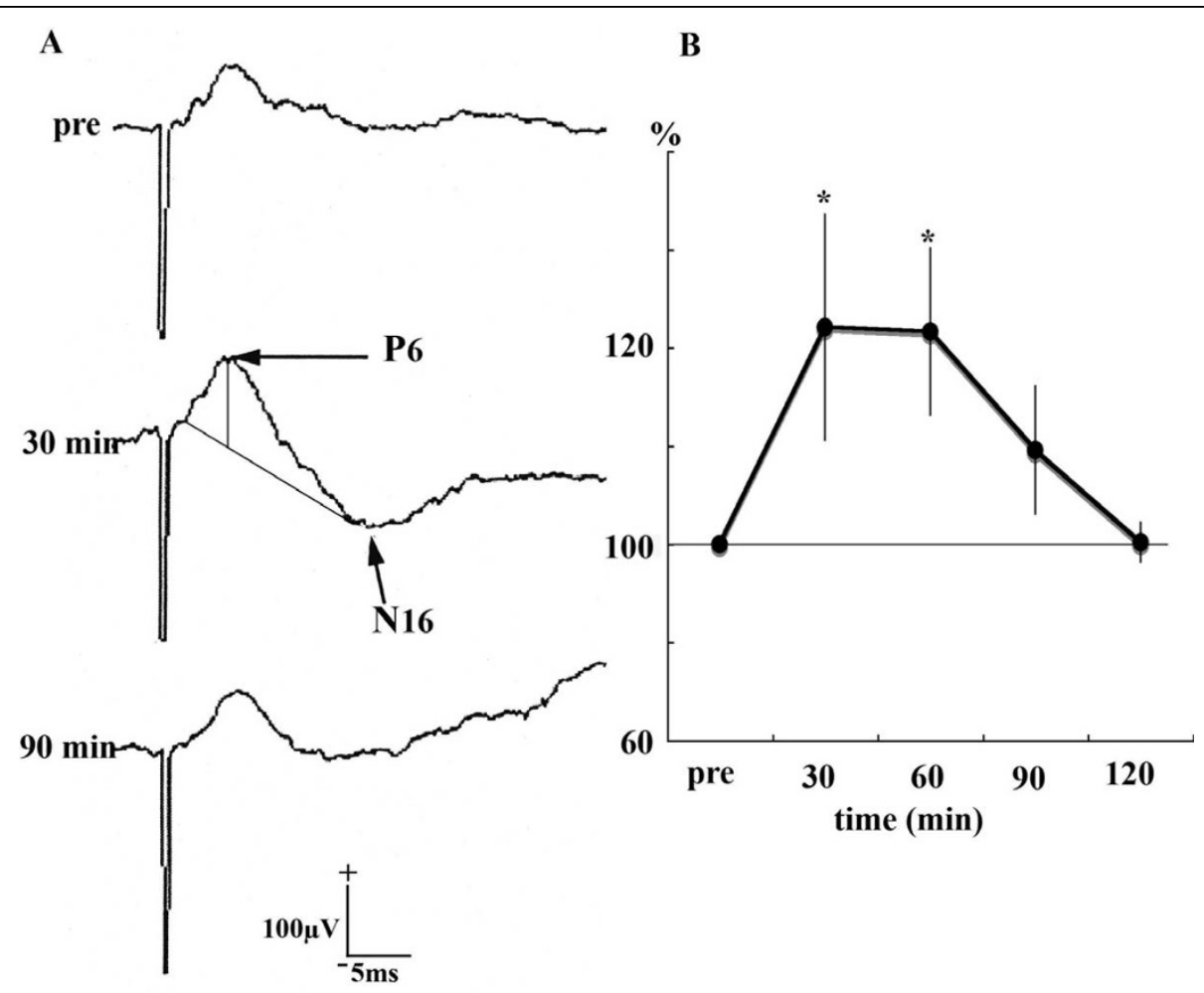

Figure 3 Field potentials evoked by CA1/subiculum stimulation. A: A field potential recorded in the PFC was composed of a positive wave with a 6 ms peak latency and a negative wave with a 16 ms peak latency. HFS delivered to CA1/SB increased the amplitude of the positive waves. B: Potentiation of field potentials started within $10 \mathrm{~min}$ after HFS and returned to the control value in approximately 90 min.

\section{Statistical Analysis}

Significant differences in discharges evoked by mechanical stimuli were assessed with the nonparametric pairedtest (Wilcoxon) to compare pre- and post-stimulation values. Data are expressed as means \pm standard errors (S.E.). Results for the numbers of Fos positive cells were statistically analyzed with the Mann-Whitney test (untreated group versus HFS/LFS group). A probability level of $<0.05$ was considered significant.

\section{Results}

\section{HFS changes pain responses in the PFC}

Noxious mechanical stimulation delivered to peripheral tissue elicited unit discharges and the duration of these responses reportedly reflects stimulus intensity [27]. We measured the duration of noxious discharges to assess the intensity of responses. HFS delivered to the CA1/SB induced two types of long lasting changes in nociceptive responses in the PFC. (Fig 4A). Seventy-eight percent (21/27 animals) of nociceptive neurons showed increases in response duration while, conversely, 22\% (6/27 animals) of PFC nociceptive neurons showed decreases of response durations. The plastic changes in nociceptive responses induced by HFS were seen within $10 \mathrm{~min}$ after applying HFS and persisted for $120 \mathrm{~min}$. The plasticity processes with maximal effects at $30 \sim 40 \mathrm{~min}$ coincided with the field potential changes observed in our preliminary experiments. LTE of pain responses (40 neurons in 21 animals) was $142.7 \pm 11 \%$ versus the prestimulus control at $10 \sim 20 \min (\mathrm{p}<0.001), 163.7 \pm$ $10 \%$ at $30 \sim 40 \min (\mathrm{p}<0.0001), 130.5 \pm 7 \%$ at $60 \sim$ $70 \min (\mathrm{p}<0.001), 117.5 \pm 6.5 \%$ at $90 \sim 100 \min (\mathrm{p}<$ $0.03)$ and $97 \pm 9.3 \%$ at $120 \mathrm{~min}(\mathrm{p}=0.4)$. All recording points were located at superficial layers II/III of the cingulate and prelimbic areas (Fig 1). Unit discharges (12 neurons in 6 animals), which showed LTD, occurred mainly in the prelimbic area (Fig 1) and stimulus points were scattered throughout the CA1/SB areas. LTD of pain responses was $10.5 \pm 7 \%$ at $10 \sim 20 \mathrm{~min}$ (p < 0.02,), $8.5 \pm 3$ at $30 \sim 40 \min (\mathrm{p}<0.0001), 51 \pm 13 \%$ at $60 \sim$ $70 \min (\mathrm{p}=0.03)$ and $75 \pm 16 \%$ at $90 \sim 100 \mathrm{~min}$ $(\mathrm{p}=0.17)$.

\section{LFS induced LTD of pain responses}

LFS delivered to the CA1/SB induced LTD of nociceptive responses (10 neurons in 5 animals, Fig 4B) in the PFC. The onset of decreased pain responses was seen at approximately 250 stimulus bursts and depression gradually reached profound levels. Pain responses decreased to $33.5 \pm 11 \%$ at $10 \sim 20 \mathrm{~min}(\mathrm{p}<0.005), 49.3 \pm 11 \%$ at 
A

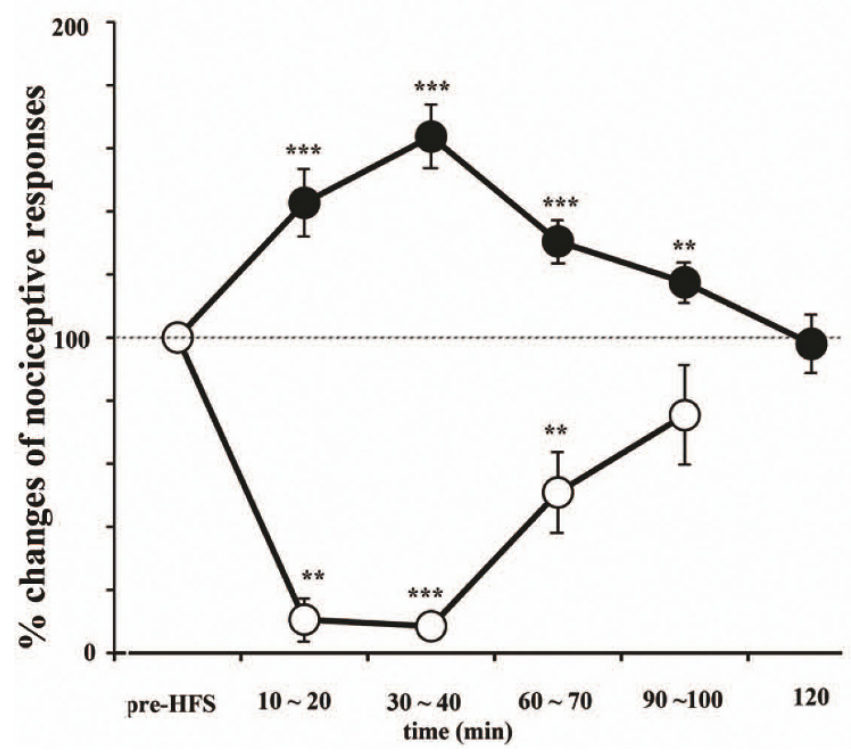

B

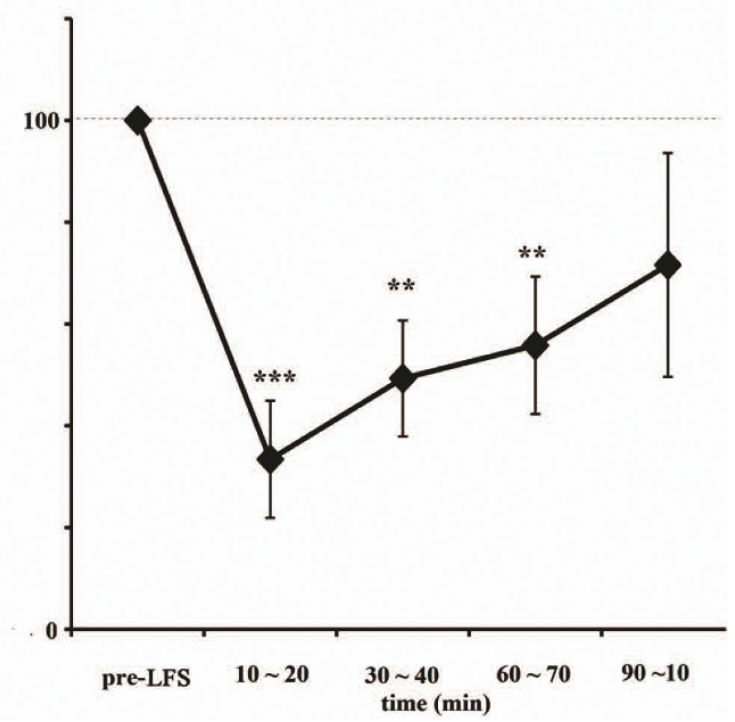

Figure $4 \mathrm{HFS} / \mathrm{LFS}$ induced LTE and LTD of nociceptive responses in the PFC A: HFS (100 Hz, $20 \mu \mathrm{A}, 15 \mathrm{~s})$ delivered to CA1/SB induced LTE (solid circles) or LTD (open circles) of nociceptive responses in the PFC. B: LFS ( $1 \mathrm{~Hz}, 5$ pulses, 600 bursts) induced LTD of nociceptive responses (solid squares). The means of three successive trials before HFS (or LFS) served as the control value (100\%). Statistical analysis: ${ }^{* *} p<0.01,{ }^{* * *} p<0.001$

$30 \sim 40 \min (\mathrm{p}<0.01), 71.6 \pm 22 \%$ at $60 \sim 70 \min (\mathrm{p}<$ 0.02 ) and then recovered to the control level by $90 \sim$ $100 \min (\mathrm{p}=0.3)$. Background spontaneous discharges decreased markedly in all cases, while, on the contrary, HFS did not affect background activities. All recording points were located at superficial layers II/III of the cingulate and prelimbic areas (Fig 1). LTD recording points were located only in the prelimbic area and the LTE of nociceptive responses were scattered throughout both the cingulate and the prelimbic areas (Fig 1). In two animals (three units), HFS was delivered to the same neurons after the nociceptive response had recovered to the pre-LFS level. After a one-hour observation period to confirm stable responses, HFS applied to CA1/SB produced LTE of nociceptive responses in the same neurons. LTE of nociceptive responses increased to $151 \%$ (mean of three neurons) of the control value at $10 \mathrm{~min}$, $230 \%$ at $30 \mathrm{~min}$ and $150 \%$ at $60 \mathrm{~min}$. The potentiation lasted $60 \mathrm{~min}$, i.e. the entire experimental period. The number of neurons was too small to allow statistical analysis, but the tendencies for LTE were very similar in these three neurons.

\section{Fos expression in the PFC after HFS or LFS}

Mean numbers of Fos positive cells in control animals were $23.2 \pm 2.2$ (left side) and 20.4 \pm 1.0 (right side) in the cingulate area and $19.7 \pm 0.9$ (left side) and $21.9 \pm$ 1.3 (right side) in the prelimbic area (Fig 5). There were no significant differences between the two sides or between the cingulate and prelimbic areas. HFS had no significant effects on Fos expression in the cingulate area (from $23.2 \pm 2.2$ to $19.6 \pm 1$ ), although HFS induced LTE of nociceptive responses. LFS significantly decreased Fos positive cells on the ipsilateral side (5.6 \pm $0.6, \mathrm{p}<0.0001$, Fig 5) but induced no changes in the contralateral side $(23.61 \pm 2.3)$ of the prelimbic area.

\section{Discussion and Conclusions}

Nociceptive information from peripheral tissue mainly projected to the superficial layers of the cingulate and prelimbic areas [27], while subiculum fibers were reportedly distributed in all layers of the prelimbic area [14]. These observations suggest that inputs from the CA1/ $\mathrm{SB}$ and nociceptive information are integrated in PFC pyramidal cells. In our experiments, HFS delivered to $\mathrm{HP} / \mathrm{SB}$ induced heterosynaptic potentiation in HP-PFC pathways (reflecting LTP of field potentials) and peripheral-PFC sensory pathways (plasticity of nociceptive responses). The HFS induced either LTE (78\%) or LTD (22\%) of nociceptive responses. Intracellular recording of PFC neurons demonstrated direct monosynaptic projections from the HP to prelimbic pyramidal cells [28], excitations of which were followed by prolonged inhibition after HP stimulation. The interneurons of the prelimbic area also receive direct projections from the HP [18]. HFS applied to HP could produce synaptic 


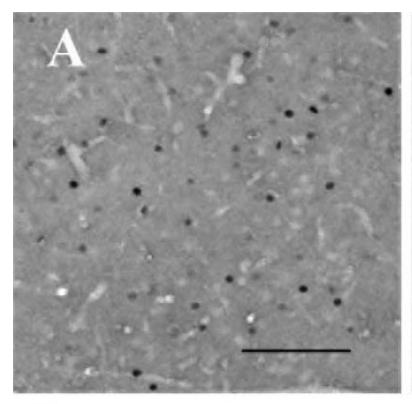

D

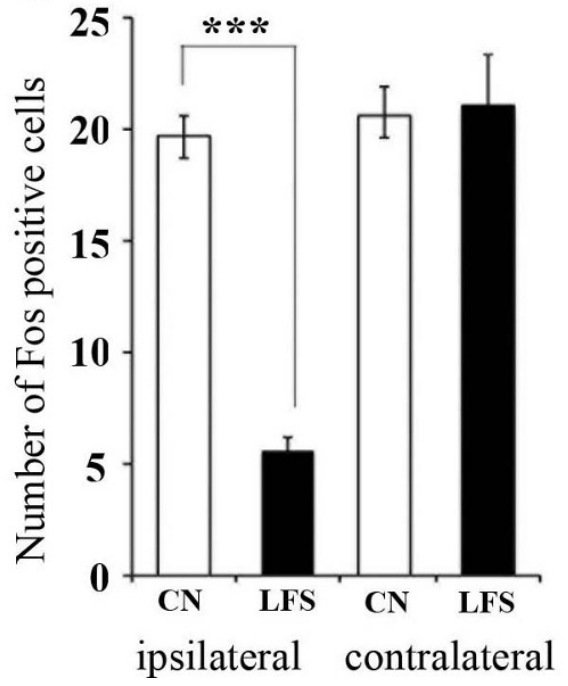

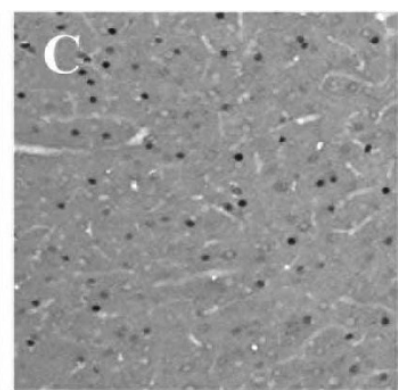

E

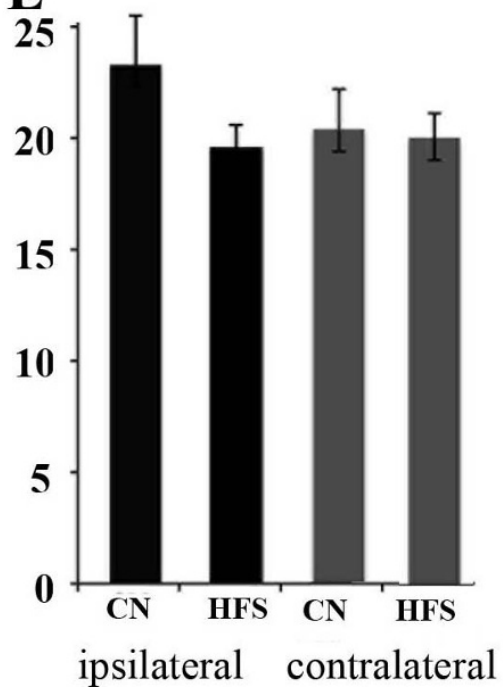

Figure 5 Fos staining in prelimbic areas of the PFC. A: An untreated control showed Fos positive cells in the prelimbic area. B: LFS significantly decreased Fos expression in the ipsilateral prelimbic area. C: An untreated control showed Fos positive cells in the cingulate area. Scale lines represent $50 \mu \mathrm{m}$. D: Numbers of Fos-expressing cells in the ipsilateral and contralateral prelimbic areas before and after LFS. Fos positive cells were decreased in the prelimbic area. CN: control, LFS: low frequency stimulation *** $P<0.001$. E: Numbers of Fos-expressing cells in the ipsilateral and contralateral cingulate areas before and after HFS.

potentiation at both pyramidal cells and interneurons, allowing HFS to induce either LTE or LTD.

NMDA mediated plasticity in the prefrontal cortex was recently reviewed [29]. NMDA receptor subtype NR2B was required for LTP of the prefrontal cortex [30], as in the hippocampus. Moreover, NMDA receptor activities were also required for induction of metabotropic glutamate receptor (mGulR) mediated LTD [31] in the perirhinal cortex. PFC neurons showed induction of LTD with NMDA receptor activities and presynaptic stimulation [32]. Postsynaptic mechanisms may have been responsible for the heterosynaptic plasticity observed in our study. In slice studies, moreover, LTP and LTD could be induced under the same stimulus conditions, depending on the dopamine concentration [33]. Postsynaptic dopamine receptor D1 activation reportedly enhances NMDA mediated-excitatory post-synaptic currents [34] and increases surface expression of NMDA receptors in pyramidal cells [35]. Our experiments were performed in vivo, such that dopamine levels may have varied. Further experiments are necessary to elucidate the synaptic mechanisms underlying the plasticity of nociceptive responses.

LFS delivered to $\mathrm{HP} / \mathrm{SB}$ induced LTD in nociceptive responses to peripheral noxious stimuli. LFS (1-5 Hz, 300 600 pulses) produces LTD mediated by the glutamate receptor, NMDA [36] and mGulR [37] in the HP. In HP-PFC pathways, a train of $1 \mathrm{~Hz}$ bursts of stimulation applied to CA1/SB regions induced LTD of field potentials [20], while HFS produced LTP. HP-PFC pathways commonly expressed bidirectional synaptic plasticity in response to different stimuli [38]. Our results, obtained from only two animals, also showed bidirectional plasticity of nociceptive discharges. Earlier studies demonstrated homosynaptic bidirectional plasticity in the PFC, while the plasticity of nociceptive responses in our experiments was heterosynaptically induced.

LFS significantly decreased Fos expression in cells in the ipsilateral prelimbic area. The histological data were consistent with the areas in which LTD was recorded in 
the electrophysiological experiments. LFS delivered to $\mathrm{HP} / \mathrm{SB}$ may inhibit excitation of prelimbic pyramidal cells via HP-PFC pathways. Thus, LFS decreased Fos expression and induced LTD of nociceptive responses.

The PFC is the center of the affectional dimension of pain and involves memories of fear, which are formed by pain experiences. Strong sensory information from peripheral nerves, such as the effects of amputationinduced LTP on synapses receiving information from sensory nerves, may be the cause of phantom pain [39]. Connections between the HP and PFC have been suggested to participate in learning and memory [40]. We established that the plasticity of nociceptive responses recorded in the PFC was produced by HFS/LFS applied to the CA1/SB. The affectional dimension of pain involves memories of fear, which are formed by pain experiences. Our results suggest HP-PFC pathways to be involved in affectional memory in pain processing.

\section{Authors' contributions}

All three authors participated in the preparation of this manuscript. and approved the final manuscript. The individual contributions of three authors to the manuscript are below.

H Nakamura carried out the electrophysiological studies and statistical analysis.

Y Katayama carried out the immunostaining and statistical analysis of Fos expression.

Y Kawakami conceived the study and coordinated all experiments.

Received: 18 March 2010 Accepted: 17 August 2010

Published: 17 August 2010

\section{References}

1. Rainville P, Duncan G, Price D, Carrier B, Bushnell M: Pain affect encoded in human anterior cingulate but not somatosensory cortex. Science 1997, 277(5328):968-971.

2. Sawamoto N, Honda M, Okada T, Hanakawa T, Kanda M, Fukuyama H, Konishi J, Shibasaki H: Expectation of pain enhances responses to nonpainful somatosensory stimulation in the anterior cingulate cortex and parietal operculum/posterior insula: an event-related functional magnetic resonance imaging study. J Neurosci 2000, 20(19):7438-7445.

3. Singer T, Seymour B, O'Doherty J, Kaube H, Dolan R, Frith C: Empathy for pain involves the affective but not sensory components of pain. Science 2004, 303(5661):1157-1162

4. Gao Y, Ren W, Zhang Y, Zhao Z: Contributions of the anterior cingulate cortex and amygdala to pain- and fear-conditioned place avoidance in rats. Pain 2004, 110(1-2):343-353.

5. Vogt BAVL: Anterior cingulate cortex and the medial pain system. In Neurobiology of cingulated cortex and limbic thalamus. Edited by: Vogt BA, Gabriel M. Boston: Birkhauser; 1993:313-344.

6. Jung M, Baeg E, Kim M, Kim Y, Kim J: Plasticity and memory in the prefrontal cortex. Rev Neurosci 2008, 19(1):29-46.

7. Hirsch J, Crepel F: Use-dependent changes in synaptic efficacy in rat prefrontal neurons in vitro. J Physiol 1990, 427:31-49.

8. Jay T, Burette F, Laroche S: NMDA receptor-dependent long-term potentiation in the hippocampal afferent fiber system to the prefrontal cortex in the rat. Eur J Neurosci 1995, 7(2):247-250.

9. Wei F, Xu Z, Qu Z, Milbrandt J, Zhuo M: Role of EGR1 in hippocampal synaptic enhancement induced by tetanic stimulation and amputation. $J$ Cell Biol 2000, 149(7):1325-1334.

10. Khanna S, Sinclair J: Noxious stimuli produce prolonged changes in the CA1 region of the rat hippocampus. Pain 1989, 39(3):337-343.
11. Bingel U, Quante M, Knab R, Bromm B, Weiller C, Büchel C: Subcortical structures involved in pain processing: evidence from single-trial fMRI. Pain 2002, 99(1-2):313-321.

12. Ploghaus A, Narain C, Beckmann C, Clare S, Bantick S, Wise R, Matthews $P$, Rawlins J, Tracey I: Exacerbation of pain by anxiety is associated with activity in a hippocampal network. J Neurosci 2001, 21(24):9896-9903.

13. Swanson L: A direct projection from Ammon's horn to prefrontal cortex in the rat. Brain Res 1981, 217(1):150-154.

14. Jay $\mathrm{T}$, Witter M: Distribution of hippocampal CA1 and subicular efferents in the prefrontal cortex of the rat studied by means of anterograde transport of Phaseolus vulgaris-leucoagglutinin. J Comp Neurol 1991, 313(4):574-586.

15. Ferino F, Thierry A, Glowinski J: Anatomical and electrophysiological evidence for a direct projection from Ammon's horn to the medial prefrontal cortex in the rat. Exp Brain Res 1987, 65(2):421-426.

16. Carr D, Sesack S: Hippocampal afferents to the rat prefrontal cortex: synaptic targets and relation to dopamine terminals. J Comp Neurol 1996, 369(1):1-15.

17. Laroche $S$, Jay $T$, Thierry A: Long-term potentiation in the prefrontal cortex following stimulation of the hippocampal CA1/subicular region. Neurosci Lett 1990, 114(2):184-190.

18. Tierney $P$, Dégenètais $E$, Thierry A, Glowinski J, Gioanni Y: Influence of the hippocampus on interneurons of the rat prefrontal cortex. Eur $J$ Neurosci 2004, 20(2):514-524.

19. Jay T, Glowinski J, Thierry A: Selectivity of the hippocampal projection to the prelimbic area of the prefrontal cortex in the rat. Brain Res 1989, 505(2):337-340.

20. Takita M, Izaki Y, Jay T, Kaneko H, Suzuki S: Induction of stable long-term depression in vivo in the hippocampal-prefrontal cortex pathway. Eur J Neurosci 1999, 11(11):4145-4148.

21. Finch DM: Hippocampal, suibcular and entorhinal afferents and synaptic integration in rodent cingulate cortex. In Neurobiology of cingulate cortex and limbic thalamus. Edited by: Vogt BA, Gabriel M. Boston: Birkhauser; 1993:.

22. Thierry A, Gioanni Y, Dégénétais E, Glowinski J: Hippocampo-prefrontal cortex pathway: anatomical and electrophysiological characteristics. Hippocampus 2000, 10(4):411-419.

23. Wang G, Cai J: Reversible disconnection of the hippocampal-prelimbic cortical circuit impairs spatial learning but not passive avoidance learning in rats. Neurobiol Learn Mem 2008, 90(2):365-373.

24. Nagata T, Suzuki H, Zhang R, Ozaki M, Kawakami Y: Mechanical stimulation activates small fiber mediated nociceptive responses in the nucleus gigantocellularis. Exp Brain Res 2003, 149(4):505-511.

25. Jay T, Burette F, Laroche S: Plasticity of the hippocampal-prefrontal cortex synapses. J Physiol Paris 1996, 90(56):361-366.

26. Paxinos G, Watson C: The Rat Brain in Stereotaxic Coordinates. San Diego:: Academic Press, 41998.

27. Zhang R, Tomida M, Katayama Y, Kawakami Y: Response durations encode nociceptive stimulus intensity in the rat medial prefrontal cortex. Neuroscience 2004, 125(3):777-785.

28. Dégenètais $E$, Thierry A, Glowinski J, Gioanni Y: Synaptic influence of hippocampus on pyramidal cells of the rat prefrontal cortex: an in vivo intracellular recording study. Cereb Cortex 2003, 13(7):782-792.

29. Zhuo M: Cortical excitation and chronic pain. Trends Neurosci 2008, 31:199-207.

30. Zhao MG, Toyoda H, Lee Y, Wu L, Ko S, Zhang X, Jia Y, Shum F, Xu H, Li B, et al: Roles of NMDA NR2B subtype receptor in prefrontal long-term potentiation and contextual fear memory. Neuron 2005, 47(6):859-872.

31. Cho K, Kemp N, Noel J, Aggleton JP, Brown MW, Bashir Zl: A new form of long-term depression in the perirhinal cortex. Nature Neuroscience 2000, 3(2):150-156.

32. Toyoda H, Zhao M, Zhuo M: Roles of NMDA receptor NR2A and NR2B subtypes for long-term depression in the anterior cingulate cortex. Eur J Neurosci 2005, 22(2):485-494.

33. Blond $\mathrm{O}$, Crépel $\mathrm{F}$, Otani S: Long-term potentiation in rat prefrontal slices facilitated by phased application of dopamine. Eur J Pharmacol 2002, 438(1-2):115-116.

34. Gonzalez-Islas C, Hablitz J: Dopamine enhances EPSCs in layer II-III pyramidal neurons in rat prefrontal cortex. J Neurosci 2003, 23(3):867-875. 
35. Gao C, Wolf M: Dopamine receptors regulate NMDA receptor surface expression in prefrontal cortex neurons. J Neurochem 2008, 106(6):2489-2501.

36. Cheong M, Yun S, Mook-Jung I, Kang Y, Jung M: Induction of homosynaptic long-term depression in entorhinal cortex. Brain Res 2002, 954(2):308-310.

37. Manahan-Vaughan D: Group 1 and 2 metabotropic glutamate receptors play differential roles in hippocampal long-term depression and longterm potentiation in freely moving rats. J Neurosci 1997, 17(9):3303-3311.

38. Izaki Y, Takita M, Akema T: Compatibility of bidirectional synaptic plasticity on hippocampo-prefrontal cortex pathway in rats. Neurosci Lett 2003, 345(1):69-71.

39. Wei F, Zhuo M: Potentiation of sensory responses in the anterior cingulate cortex following digit amputation in the anaesthetised rat. J Physiol 2001, 532(Pt 3):823-833.

40. Laroche S, Davis S, Jay T: Plasticity at hippocampal to prefrontal cortex synapses: dual roles in working memory and consolidation. Hippocampus 2000, 10(4):438-446.

doi:10.1186/1471-2202-11-100

Cite this article as: Nakamura et al: Hippocampal CA1/subiculumprefrontal cortical pathways induce plastic changes of nociceptive responses in cingulate and prelimbic areas. BMC Neuroscience 2010 11:100.

\section{Submit your next manuscript to BioMed Central} and take full advantage of:

- Convenient online submission

- Thorough peer review

- No space constraints or color figure charges

- Immediate publication on acceptance

- Inclusion in PubMed, CAS, Scopus and Google Scholar

- Research which is freely available for redistribution

Submit your manuscript at www.biomedcentral.com/submit 